\title{
Document Identifier with Attribute Identifier
}

National Cancer Institute

\section{Source}

National Cancer Institute. Document Identifier with Attribute Identifier. NCI Thesaurus.

Code C93809.

A unique symbol that establishes identity of the document. 